\title{
Interconnection and Simulation Issues in Haptics
}

\author{
Gianni Borghesan, Member, IEEE, Alessandro Macchelli, Member, IEEE, Claudio Melchiorri, Senior \\ Member, IEEE
}

\begin{abstract}
In this paper, three results are presented concerning certain computational/control aspects crucial for the proper behavior of haptic devices. The first one is a novel technique for a real-time simulation of virtual environments that is able to preserve the energetic behavior of the simulated physical system and to avoid undesired effects related to unstable behaviors of the haptic device. The proposed real-time integration method is simpler, in terms of computational complexity, than similar solutions known in the literature, and provides an additional insight when "faulty conditions" are met. Secondly, a new method for the energy-consistent interconnection of discrete-time physical systems, implemented by algorithms running at different frequencies (i.e. multi-rate systems), is illustrated. Multi-rate systems are very common in haptics, since the frequency at which the control law of the haptic interface is executed is usually higher than the frequency of the simulation of the virtual environment. Finally, the third result presented in this paper concerns the problem of energy generation due to the time discretization in the acquisition of the haptic interface position. Similarly to the previous case, a technique for an energy-consistent analog/digital conversion is proposed. All these methodologies have been validated both by simulations and experiments.
\end{abstract}

Index Terms—haptics, passivity, real-time simulation, multi-rate systems, port-Hamiltonian systems

\section{INTRODUCTION}

$\mathrm{O}$ $\mathrm{NE}$ of the main goals for haptic systems is to achieve a "realistic" interaction with virtual environments, i.e. the haptic rendering of a simulated physical object should emulate "perfectly" the interaction with the real object. Haptic devices and their controllers usually have an admittance and an impedance causality respectively: the controller input is the position/velocity of the haptic interface, while the force computed by the controller is the input for the haptic device. Thus, performance of haptic interfaces can be measured in terms of the maximum stiffness achievable while rendering a virtual wall, [1]. On the other hand, as discussed in [2], there are two main aspects that may generate instability in the system, limiting the maximum stiffness and therefore reducing the achievable performance. As a matter of fact, a fictitious power generation may be caused both by time discretization, in case of multi-rate systems, or by position quantization in the analog/digital conversion, which may produce limit cycles with an amplitude proportional to the encoder resolution.

Another important issue in haptics is the multi-rate execution of different control or simulation tasks. While for simple virtual scenes the force displayed by the haptic interface is directly calculated by the software which simulates the virtual objects, in case of complex environments the computations are usually divided into two main tasks. The first task computes the force that has to be applied by the haptic interface, while the

- Preliminary versions of this work has been presented at the 2007 and 2008 IEEE International Conferences on Robotics and Automation.

- G. Borghesan (gianni.borghesan@unibo.it), A. Macchelli (alessandro.macchelli@unibo.it) and C. Melchiorri (claudio.melchiorri@unibo.it) are with the University of Bologna, Department of Electronics, Computer Science and Systems (DEIS), viale Risorgimento 2, 40136 Bologna, Italy.

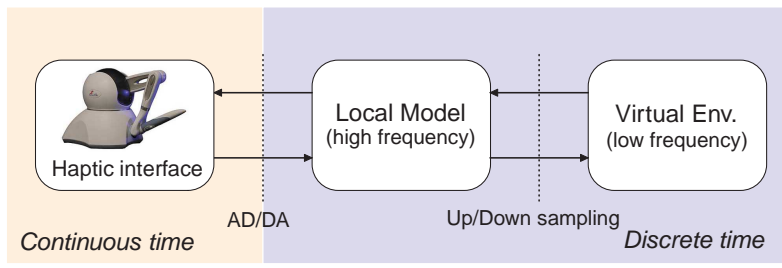

Fig. 1. Typical control architecture of a haptic interface.

second one computes the dynamics of the virtual environment. Typically, this latter task is based on local model methods that consider a simplified version of the virtual environment in the neighborhood of the position of the haptic interface, [3]. Since performance decreases as the force feedback is computed less frequently, the local model must run at high frequency and, in order to reduce the computational costs, this leads to the design of multi-rate systems. In such systems, the virtual environment is a low-frequency task, which carries out the heavy computations associated with the dynamics of complex systems, while the local model is a highfrequency process which assures stability and high performance. A schematic representation of a multi-rate control architecture is shown in Fig. 1.

The stability of single- and multi-rate systems is typically studied with two different approaches. With the first one, the system can be treated as a whole and conclusions on its stability in closed-loop can be drawn either in the time or in the frequency domain by studying the range of variation of critical parameters (e.g., the virtual wall stiffness), [1], [2], [4], [5], [6], or by considering the relation between local model and virtual environment stiffness in multi-rate systems, [7], [8]. The second approach relies on considering the system as the (power conserving) interconnection of simpler sub-systems. In 
this way, the stability of the whole system follows once the passivity of each sub-system has been proved and/or forced by means of suitable control actions. This methodology has been developed in [9], [10], where the main sub-systems are the haptic interface and the digital control (including the analog/digital conversion), and in [11], [12], [13], [14] where also the digital controller is considered as composed by lower complexity sub-systems, designed to be intrinsically passive. Both methods either explicitly or implicitly rely on passivity. Passivity-based methods are appealing in haptics since they do not explicitly take into account the presence in closed-loop of the human operator, whose behavior is also very difficult to be formulated in an analytic manner. The assumption that the passivity of the system composed by virtual environment and haptic interface is sufficient for the stability of the whole system (i.e., with the human operator), is based on the hypothesis that the user, who is not a passive "system" (in fact, he can indefinitely inject energy via the haptic interface to destabilize the system), behaves in a "collaborative manner", i.e. without using more energy than in everyday life for carrying out the task. Moreover, it is worth mentioning that human operators are used to interact with passive objects, so a realistic haptic rendering requires that the virtual objects retain the passivity properties of real objects.

In this paper, the above problems have been tackled within the port-Hamiltonian and passivity frameworks. At first, since the simulation of a dynamic system must preserve its passivity properties, a novel real-time and physically consistent integration method is discussed. A preliminary version has been presented in [15], but here it has been improved and validated by means of both simulations and laboratory experiments. It is based on the general theory of sampled port-Hamiltonian systems [13], and its main feature is that the state update criterion considers both the continuous time dynamics and its energy properties, so that the passivity properties of the continuous-time system are maintained even at low frequencies and/or large integration steps. Originally introduced in [11] for first-order systems, here this algorithm is generalized to consider real-time simulation of higher-order dynamical systems.

In Sect. 4.1 the problem of the passive interconnection of two discrete-time systems characterized by different sampling frequencies is considered. Due to the fact that, in multi-rate systems, energy is artificially generated by the up/down sampling operations, a novel "component", the Passive Sample and Hold (PSH), is introduced. This element has been realized by following the same rationale of the Passivity Observer/Passivity Controller, [9]. In fact, the PSH controls the power exchange between the two systems and reduces the energy error by acting on one of the port signals (velocity/force). However, if compared to the standard Passivity Observer/Passivity Controller, performance has been improved by limiting the amount of (virtually) generated energy that can be dissipated in a integration step, thus bounding the maximum allowable correction on the velocity or force signal.

The third main result of this paper is presented in Sect. 4.2, where a novel solution for the problem of passively interconnecting a continuous-time system with a discrete-time one is illustrated. In this case, the continuous-time system is the haptic interface, while the discrete-time one is the controller. The data exchanged between the two systems are position/velocity and force information. Passivity can be preserved by introducing the element here called as the Passive Continuous Discrete Time Connector. Similarly to the PSH, this element keeps track of the energy error generated by discretization of the joint positions of the haptic device (and by the following estimation of the joint velocities), and consequently modifies the velocity signal to be passed to the controller in order to reduce the energy error. Differently from [16], a bound on the maximum dissipated energy in a sampling period is computed online, in order to avoid large and sudden corrections of the velocity signal fed to the virtual environment, obtaining a smoother interaction while preserving passivity. The proposed algorithms have been validated with the help of extensive simulation and experimental activities, reported in Sect. 5 and 6 respectively. Conclusions and final remarks are discussed in Sect. 7.

\section{BACKGROUND}

\subsection{Continuous- and discrete-time port-Hamiltonian systems}

In this paper the haptic interface, the virtual environment, and the local model are modeled as portHamiltonian systems [17], [18], [19], [20] because this formalism is deeply connected with passivity theory and allows the definition of complex systems as the result of the power conserving interconnection of simpler components that exchange energy through their power ports. A port-Hamiltonian system is described by:

$$
\begin{aligned}
\dot{x} & =[J(x)-R(x)] \frac{\partial \mathcal{H}}{\partial x}+G(x) u \\
y & =G^{T}(x) \frac{\partial \mathcal{H}}{\partial x}
\end{aligned}
$$

and satisfies

$$
\frac{\mathrm{d} \mathcal{H}}{\mathrm{d} t}=-\frac{\partial^{T} \mathcal{H}}{\partial x} R(x) \frac{\partial \mathcal{H}}{\partial x}+y^{T} u
$$

where (1a) and (1b) define respectively the state update law and the output, while (2) is the power balance equation. Here, $x \in \mathbb{R}^{n}$ denotes the state variable, $J(x)=$ $-J^{T}(x)$ is the interconnection matrix, $R(x)=R^{T}(x) \geq 0$ the dissipation matrix, $G(x)$ the input-output matrix and $\mathcal{H}(x)$ is the Hamiltonian function, i.e. the total energy of the system. The pair of signals $(u, y)$ defines the power port, and $y^{T} u$ is the associated power flow. For example, in mechanical systems, input-output signals are velocities and forces; (generalized) forces are referred to 


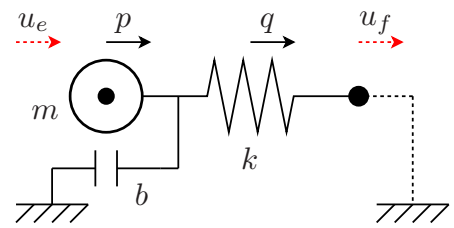

Fig. 2. A mass-spring-damper system.

as efforts, denoted by $e$, while (generalized) velocities are flows, and are denoted by $f$. A port-Hamiltonian system is passive iff the Hamiltonian function is lower-bounded, i.e. iff $\mathcal{H}(x) \geq \mathcal{H}_{\min }, \forall x \in \mathbb{R}^{n}$. This means that the system can provide, at most, its initial energy, [19].

Example 1: The mass-spring-damper device of Fig. 2 can be easily written in port-Hamiltonian form:

$$
\begin{aligned}
& \dot{x}=\left[\begin{array}{l}
\dot{q} \\
\dot{p}
\end{array}\right]=\left\{\left[\begin{array}{cc}
0 & -1 \\
1 & 0
\end{array}\right]-\left[\begin{array}{ll}
0 & 0 \\
0 & b
\end{array}\right]\right\}\left[\begin{array}{c}
k q \\
p / m
\end{array}\right]+G u \\
& y=G^{T}\left[\begin{array}{c}
k q \\
p / m
\end{array}\right]
\end{aligned}
$$

where the energy function is

$$
\mathcal{H}(x)=\frac{1}{2}\left(k q^{2}+\frac{p^{2}}{m}\right)
$$

The state is $x=[q, p]^{T}$, where $q$ and $p$ are the spring elongation and mass momentum respectively. Moreover, $k$ is the spring stiffness, $b$ the damping coefficient, and $m$ the mass. Now, denote by $u_{f}$ the velocity of the right side of the spring and by $u_{e}$ the force acting on the mass. If $u=\left[u_{f}, u_{e}\right]^{T}$, then $G$ is the identity matrix and the system has two outputs (the spring force $k q$ and the mass velocity $p / m$ ). On the other hand, if the right side of the spring is interconnected to ground, the system clearly has only one input $u=u_{e}$ and one output, the mass velocity $p / m$, with $G=\left[\begin{array}{ll}0 & 1\end{array}\right]^{T}$. In the following, when the mass-spring-damper system is considered, we implicitly refer to this latter case.

\subsection{Real time simulation of continuous time systems}

A common approach for the real time simulation of dynamical systems is based on the forward Euler integration method, mostly because it is easy to implement and presents a low computational burden. The continuous time system (1) is, then, discretized as follows:

$$
\begin{aligned}
\Delta x(k) & =\left.[J(x)-R(x)] \frac{\partial \mathcal{H}}{\partial x}\right|_{x=x(k)}+G(x(k)) u(k) \\
x(k+1) & =x(k)+T \Delta x(k) \\
y(k) & =\left.G^{T}(x(k)) \frac{\partial \mathcal{H}}{\partial x}\right|_{x=x(k)}
\end{aligned}
$$

where $x(k)$ is the discrete-time state and $u(k)$ the input at $t=k T$, with $T$ the fixed simulation step. This algorithm does not assure the passivity or the stability of the discrete-time system, as shown in the next example.

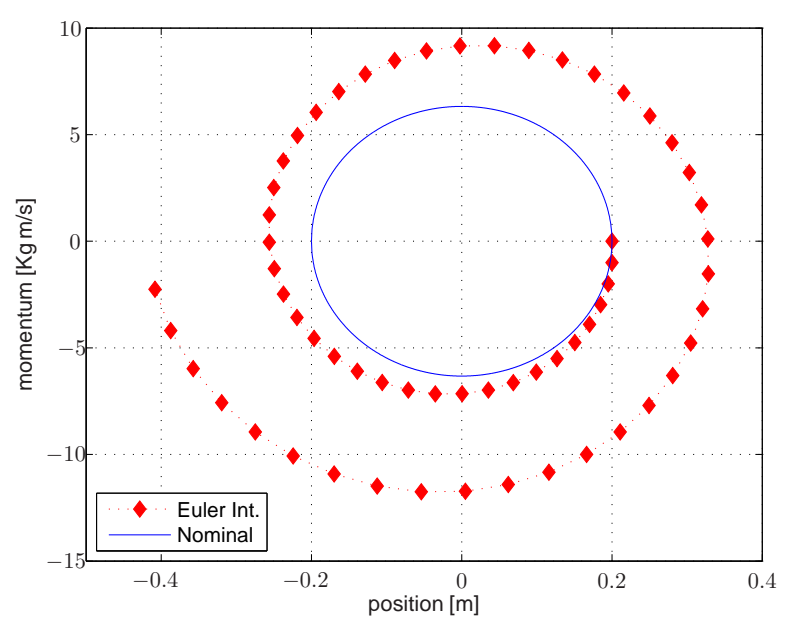

Fig. 3. Phase diagram of a mass-spring system. The solid line is the trajectory of the continuous time system, while diamonds are the states of the discretized system.

Example 2: Consider the mass-spring system of Example 1, with $k=5000 \mathrm{~N} / \mathrm{m}, m=0.2 \mathrm{~kg}, b=0 \mathrm{~N} \cdot \mathrm{s} / \mathrm{m}$, $q(0)=0.2 \mathrm{~m}$ and $p(0)=0 \mathrm{~kg} \cdot \mathrm{m} / \mathrm{s}$ and assume that $u_{f}=u_{e}=0$. Since no dissipation is present and the system is autonomous, energy is conserved and the state evolves in a set of iso-energetic states that depends on the initial conditions. The state evolution, in the state space, is reported in Fig. 3 with a solid line, while a dashed line denotes the state evolution of (5), with the same initial state and parameters, and an integration step $T=1 \mathrm{~ms}$. Note that the discrete-time system is characterized by an unstable behavior, and therefore passivity is not preserved in discrete-time. The same result can be formally deduced in the $\mathcal{Z}$-transform domain by studying the discrete time system, where the continuous time integrators (mass and spring) are replaced by their discrete counterparts, i.e. $1 / s \rightarrow \frac{T}{z-1}$. On the other hand, some implicit integration methods preserve passivity, at least in the linear case, [21]. One of these is the trapezoidal method, whose mapping is defined as $s \rightarrow \frac{2(z-1)}{T(z+1)}$. The main problem that prevents the application of this or similar methods in haptic applications is their implicit nature: each system discretized by an implicit transformation results in a non strictly proper system, which is not suitable to be interconnected to a similar system because of the presence of algebraic loops, or used as a haptic interface controller. In this case, in fact, it is necessary to compute the controller state and its output in a negligible time with respect to the controller time step, since a direct effect of the input on the output is present.

\section{PASSIVITY IN NUMERICAL SIMULATIONS 3.1 Energy-consistent simulation}

In this section, the problem of implementing energyconsistent simulations of port-Hamiltonian systems is addressed, [14]. Passivity can be preserved if and only 
if at each sample time a power (or energy) balance condition is satisfied. If $H(k)$ denotes the energy of the discrete-time system at $t=k T$, and $\Delta H(k)$ its variation in the time step $k T \rightarrow(k+1) T$, clearly

$$
H(k+1)=H(k)+\Delta H(k)
$$

For port-Hamiltonian systems, the energy variation in a time step follows from the time integration of the powerbalance relation (2). In fact, if $u(t)=u(k T) \equiv u(k), y(t)=$ $y(k T) \equiv y(k)$ and $x(t)=x(k T) \equiv x(k)$ for all $t \in[k T,(k+$ 1) $T)$, then

$$
\Delta H(k)=T\left[y(k) u(k)-\frac{\partial^{T} \mathcal{H}}{\partial x} R(x) \frac{\partial \mathcal{H}}{\partial x}\right]_{x=x(k)}
$$

Note 1: $H(k)$, the energy of the discrete-time system defined by (6) and (7), may differ from $\mathcal{H}(x)$, the energy of the continuous-time system, in case some of the fault conditions described below, in Sect. 3.2, occur.

Passivity is preserved if $x(k+1)$ is consistent with the value of $H(k+1)$. Let $I_{k+1}$ be the set of eligible states whose associated energy $\mathcal{H}(x)$ is equal to $H(k+1)$, i.e.:

$$
I_{k+1}=\left\{x \in \mathbb{R}^{n} \mid \mathcal{H}(x)=H(k)+\Delta H(k)\right\}
$$

Consequently, instead of using (5), $x(k+1)$ and $y(k)$ should be computed by means of the following algorithm.

\section{Algorithm 1 (State update):}

1) Given $x(k)$ and $u(k)$, compute $\partial \mathcal{H} / \partial x$ at $t=k T$, and then $y(k)$ from $(5 c)$;

2) The energy variation $\Delta H(k)$ follows from (7);

3) The energy stored at $t=(k+1) T$, i.e. $H(k+1)$, is obtained with (6), and defines the set of eligible states $I_{k+1}$;

4) $x(k+1)$ is selected in $I_{k+1}$ according to a proper update strategy, discussed in the remaining part of this section.

The key point in Algorithm 1 is the update strategy, i.e. the selection of $x(k+1)$ in $I_{k+1}$ so that the dynamic behaviors of the discrete and continuous time systems are as close as possible. Roughly speaking, in [11] the state $x(k+1)$ was selected along the direction of the state time derivative, evaluated at $x=x(k)$ :

$$
x(k+1) \in\{x(k)+\lambda \Delta x(k), \lambda \in \mathbb{R}\}
$$

with $\Delta x(k)$ given by (5a). This method is suitable for one-dimensional systems, i.e. systems with $x \in \mathbb{R}$, where $x(k+1)$ can assume, at most, two distinct values, as illustrated in the following example.

Example 3: Consider a system with one state variable only, such as a spring or a mass. Being that $H(k+1)$ is a quadratic function, if it is non-negative there are only two admissible choices for $x(k+1)$, see Fig. 4 . In this case, since $x(k)$ is known, the next state can be defined as the "closest" one to $x(k)$. In case of ambiguity, the sign of the state derivative given by (1a) can be also taken into account. The update strategy is shown in Fig. 4.

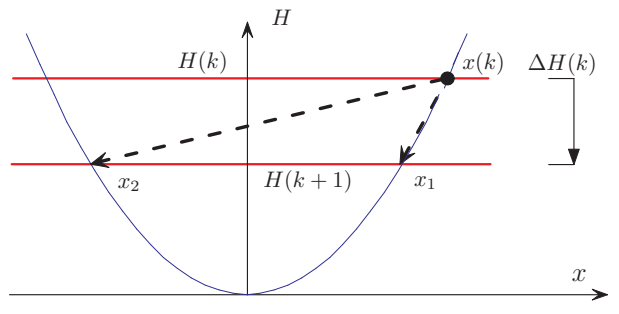

Fig. 4. State update strategy for systems with one state variable. Given $x(k)$ and $H(k+1)$, the set of eligible states at time $t=(k+1) T$ is $I_{k+1}=\left\{x_{1}, x_{2}\right\}$ and $x(k+1)=x_{1}$.

Generally speaking, in systems with more state variables, moving along the direction of the derivative of the state (see (9)) does not assure that a solution, i.e. a unique $x(k+1)$, can be found, as discussed in Examples 4 and 5, below. In fact, infinite admissible choices of $x(k+1)$ are usually available and, in principle, several state update strategies can be developed. In the remaining part of this section, a novel approach which relies on the employment of both the level curves and the field lines is presented. It is well-known that a level curve corresponds to a set iso-energetic states defined by (8), while a field line is the solution, for a given initial state, of the following differential equation:

$$
\frac{\mathrm{d} x}{\mathrm{~d} s}(s)=\frac{\partial \mathcal{H}}{\partial x}(x(s))
$$

where $s \in \mathbb{R}$ is an independent variable that parametrizes the field line.

Systems characterized by two or more state variables generally present sets of iso-energetic states of infinite cardinality. For simplicity, only linear systems are considered here, since their energy function has only one minimum and no maximum or saddle point. As a consequence, all the field lines intersect all the level curves and, as it will be clearer later on, this property is fundamental for proving the convergence of the proposed state update algorithm. It is worth mentioning, however, that similar results hold also for non-linear systems for which this condition is satisfied, for example for systems whose energy functions are radially monotonically increasing from the minimum point. As already pointed out, the next state $x(k+1)$ must be selected in the set $I_{k+1}$. On the other hand, starting from $x(k)$, there are at least two "natural" update strategies, i.e. to select $x(k+1)$ along the field lines, or along the direction of $\dot{x}$. Possible drawbacks of both these choices are illustrated in the following examples.

Example 4: Consider the mass-spring system of Example 2 with initial state $x(0)=\left[q_{0}, 0\right]^{T}$ and no active force (no input and no damping). Clearly, $H(k)=H(0)=$ $\mathcal{H}(x(0)), I_{k}=I_{0}$ and $x(k) \in I_{0}, \forall k \geq 0$. According to the first strategy, $x(k+1)$ is selected along the field lines, Fig. 5(a). However, since the field lines are perpendicular to the level curves, the only eligible state is $x(0)$, which is clearly not correct. On the other hand, by following the 


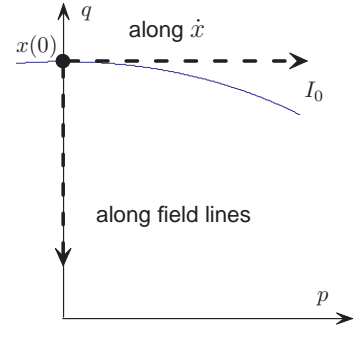

(a)

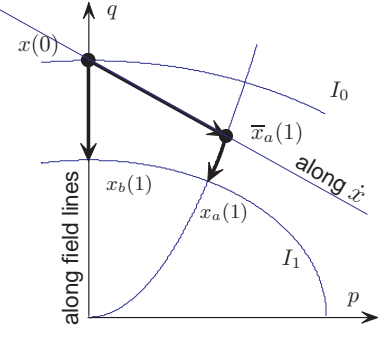

(b)
Fig. 5. Different state-update strategies for a massspring system. In the lossless case (a), moving either along the derivative or the field line does not provide a solution since there is only an intersection with $x(0)$. In (b) "some" energy is extracted: moving along the field line $x(0) \rightarrow x_{b}(1)$ results into a meaningless behavior, while along the derivative $x(0) \rightarrow x_{a}(1)$ provides no solution. The proposed method, instead, brings the state in $x(0) \rightarrow \bar{x}_{a}(1)$ with an integration step, and then looks for a solution $x_{a}(1)$ along the field line.

second strategy, the discrete state is selected as indicated by (9), i.e. by moving along the direction of $\dot{x}$, which again intersects $I_{0}$ only in $x(0)$. Therefore, as shown in Fig. 5(a), both strategies do not reproduce the behavior of the system in discrete time.

Example 5: Consider again the mass-spring system of Example 2 with the initial condition $x_{0}=\left[q_{0}, 0\right]^{T}$ and suppose that energy decreases because of an external force (e.g. generated by a damper). The state $x(1)$ must be in $I_{1}$, a set characterized by an energy level lower than $I_{0}$. If the next state is selected along the field lines (first strategy), the solution is $x_{b}(1)=\left[q_{b}, 0\right]^{T}$, Fig. 5(b), which means that a spring deformation has been generated without any velocity variation of the mass. What happens, then, is that the input defines the energetic level $I_{1}$, but it does not play any role in the selection of the state within the eligible set itself. On the other hand, if the state is chosen along $\dot{x}$ (second strategy), which depends on $u(0)$, there is no intersection with $I_{1}$, and $x(1)$, in general, cannot be determined.

In order to avoid the problems illustrated in the previous examples, a two-step procedure is introduced. At first, the traditional time integration algorithm for ODEs (e.g. forward Euler) is adopted and an intermediate state $\bar{x}_{a}(1)=x(0)+T \Delta x(0)$ is determined once $\Delta x(0)$ is computed by means of (5a). Then, starting from $\bar{x}_{a}(1)$, the next state is selected by moving towards $I_{1}$ to correct the energy error produced by the traditional integration method. Since the field lines intersect all the energy levels, if the state evolves from $\bar{x}_{a}(1)$ along this path, then the desired energy level $I_{1}$ is always reached. Note that the choice of the next state in $I_{1}$ depends on the field line "selected" by $\bar{x}_{a}(1)$, which is determined by the input $u(k)$. What happens in the situation of Example 5 is illustrated in Fig. 5(b), where it is possible to see:

- The solution obtained by moving directly along the co-vector field, i.e. $x(0) \rightarrow x_{b}(1)$;

- That there is no intersection between the direction of $\dot{x}$ and $I_{1}$

- The proposed strategy, i.e. $x(0) \rightarrow \bar{x}_{a}(1) \rightarrow x_{a}(1)$.

The intersection between the field line which includes $\bar{x}(k+1)$, and the set $I_{k+1}$ can be determined in at least three different ways:

1) When possible, the intersection is computed analytically;

2) If the explicit equation of the field lines is known, the new state can be determined by iterating a bisection method and using the energy function as cost function;

3) Otherwise, it is possible to proceed with a numerical integration of (10) (along the field lines) until $I_{k+1}$ is reached.

The first method can be used in a very limited class of problems, since the curve intersection can be computed in a closed form only for "simple" systems, such as the linear ones, for which the iso-energetic levels are "spheres" in the state space. The second method is useful in case the field line equations are known, but the intersection with the level curves cannot be computed. On the other hand, the third method is the most general and time consuming one, and can be used only if the field line equations are unknown. Note that the second and third methods introduce an "energy error" since $x(k+1)$ does not exactly belong to $I_{k+1}$. However, this error is known, bounded, and depends on the number of iterations. Consequently, it can be compensated by following the same ideas discussed in Sect. 3.2.

Note 2: The state update problem can be solved with the first method in case of linear port-Hamiltonian systems, i.e. with a quadratic energy function.

\subsection{Fault conditions}

Algorithm 1 is able to preserve the physical behavior of the system in discrete-time, i.e. it is able to keep as close to zero as possible the difference between the "real" stored energy $\mathcal{H}(x(k))$ and the "virtual" energy $H(k)$ due to discrete-time simulation. However, there are special cases in which the system cannot be updated with the proposed methodology, and in particular in the two cases of the so-called energy leap and dynamic deadlock, firstly analyzed in [11] and discussed below.

Energy leap: It may happen that, depending on the input and on the actual state of the discrete system at $t=k T$, the energy calculated for the next step is less than the minimum admissible energy, i.e. $H(k+1)=$ $H(k)+\Delta H(k)<H_{\min }$ (see Fig. 6). Then, $x(k+1)$ cannot be determined and, in this case, it is selected as an element of $I_{k}$, i.e. within the energy level at $t=k T$. This is equivalent to injecting an amount of energy equal to $|\Delta H(k)|$. In order to preserve the overall passivity, the injected energy is "logged" and then dissipated in subsequent algorithm iterations. Unfortunately, the choice of the state $x(k+1)$ can be problematic, and 


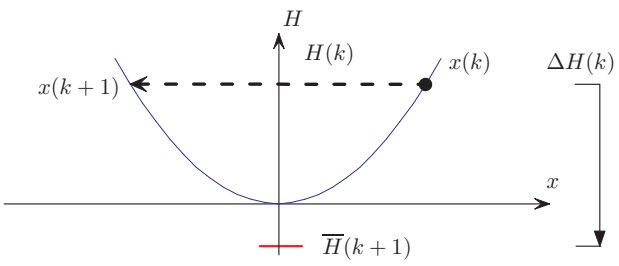

Fig. 6. Graphical representation of energy leap procedure in case of a system with one state variable. If $H(k)-\Delta H(k)<0$, an energy leap occurs: the state is updated in $x(k+1)$ and the energy error is book-kept.

depends on the number and nature of the state variables. In principle, the only constraint is to keep track of the injected energy that has to be dissipated in the next algorithm iterations. For the sake of clarity an example of energy leap, describing how the problem has been solved in [11], is reported in the following example.

Example 6: For a linear system with one state variable, when an energy leap occurs, i.e. the next energy level $\bar{H}(k+1)$ is lower than the minimum system energy, as in Fig. 6, the next state can be $x(k+1)=-x(k)$. In this way, the energy error is known and equal to $|\Delta H(x(k))|$, being $\mathcal{H}(x(k))=\mathcal{H}(x(k+1))=H(k))$.

Dynamic deadlock: This second problem occurs when the system is in correspondence to $H_{\mathrm{min}}$, the minimum of the energy function. In this case, from (1b) and (7) it is clear that, for any possible value of $u(k)$, both $y(k)$ and $\Delta H(k)$ will be equal to zero. Thus, the total energy remains unchanged and therefore the state does not evolve. Consequently, the state must be moved "artificially" by injecting a proper amount of energy.

Both situations are characterized by an energy generation effect and in the remaining part of this section a general method able to tackle these two conditions is proposed. This solution can be easily extended to other cases in which the conversion from continuous to discrete time might cause these "unnatural" effects, as discussed in Example 7. Denote with $H_{\text {new }}=H(k)+\Delta H$ the desired system energy at the next iteration step. If none of the fault conditions is met, then $H_{\text {new }}$ will be the energy at the next iteration step, i.e. $H(k+1)=H_{\text {new }}$. Otherwise, depending on the actual state, the energy function has to be modified. It is clear, however, that once the next state is chosen, also the value of $H(k+1)$ is determined. If $H_{\mathrm{BK}}(k)$ denotes the book-kept energy, i.e. the difference between the generated energy (due to energy leap and/or dynamic deadlock) and the energy already dissipated up to the $k$-th step, we have that

$$
H_{\text {new }}-H_{\mathrm{BK}}(k)=H(k+1)-H_{\mathrm{BK}}(k+1)
$$

This equation provides the value of $H_{\mathrm{BK}}$ at $t=(k+1) T$. In case of dynamic deadlock or energy leap problems, an integration step of forward Euler (or any other explicit, fixed step integration algorithm) allows the computation of the new state thanks to (5a) and (5b), while the new energy is defined as $H(k+1)=\mathcal{H}(x(k))$, and $H_{\mathrm{BK}}(k+1)$ is computed by means of (11). It is worth noting that the solution of the energy leap problem described in [11] and reported in Example 6 is a particular case of this procedure. In fact, instead of choosing the next state by means of forward Euler integration, the "symmetric" state was selected, i.e. $x(k+1)=-x(k)$. This choice leads to an unmodified energy level $H(k+1)=H(k)$. Consequently, the amount of generated energy is equal to $\Delta H(k)$, and the book-kept value must be updated accordingly, i.e. $H_{\mathrm{BK}}(k+1)=H_{\mathrm{BK}}(k)-\Delta H(k)$.

To preserve passivity, it is necessary to dissipate the amount of stored energy when dynamic deadlock and/or energy leap does not take place. A possible solution can be to dissipate an amount of energy equal to $\alpha H_{\mathrm{BK}}(k)$ during the interval $[k T,(k+1) T]$, with $0<\alpha \leq 1$. This requires that the new values of $H(k+1)$ and $H_{\mathrm{BK}}(k+1)$ must be computed as follows:

$$
\begin{aligned}
H(k+1) & =H_{\text {new }}-\alpha H_{\mathrm{BK}}(k) \\
H_{\mathrm{BK}}(k+1) & =H_{\mathrm{BK}}(k)+H(k+1)-H_{\text {new }} \\
& =(1-\alpha) H_{\mathrm{BK}}(k)
\end{aligned}
$$

Example 7: The previous method can be used for properly simulating unilateral springs. In such systems, the generated force must be positive if the spring is compressed (in case of contact), and equal to zero if not (reset condition). This second condition is useful when port-Hamiltonian systems are used in conjunction with haptic systems, since the estimation of velocity from the sampled position leads to a position drift. In this way, the synchronization between real and calculated positions can take place each time the haptic interface and the virtual environment are in contact.

Let $q(k)$ be the distance between the haptic interface and the virtual environment, and $x(k)$ the state of the spring. The unilateral spring can be implemented once the following step is "appended" to the procedure described in (12). More precisely, if $x(k+1)<0$ or $q(k)<0$ then $x(k+1)=0, H_{\mathrm{BK}}(k+1)=H_{\mathrm{BK}}(k)+H_{\text {new }}$, and $H(k+1)=0$. The algorithm is the following:

Algorithm 2 (State update, unilateral spring):

1) Given $x(k)$ and $u(k)$, compute $\partial \mathcal{H}(x) / \partial x$ at $t=k T$, the energy variation $\Delta H(k)$, and $H_{\text {new }}=H(k)+$ $\Delta H(k)$;

2) If the system is in dynamic deadlock or $H_{\text {new }}<0$, then $x(k+1)=x(k)+\Delta x T, H(k+1)=\mathcal{H}(x(k+$ $1))$ and $H_{\mathrm{BK}}(k+1)=H_{\mathrm{BK}}(k)-H_{\text {new }}$. Differently, $x(k+1)$ is computed with a proper update strategy, as discussed in Sect. 3.1;

3) If $x(k+1)<0$ or $q(k)<0$, then $x(k+1)=0$, $H_{\text {new }}=H(k), H_{\mathrm{BK}}(k+1)=H_{\mathrm{BK}}(k)+H_{\text {new }}$ and $H(k+1)=0$.

\subsection{Concluding remarks}

Even if only one dimensional examples have been presented, the extension to the more general three- or even six-dimensional case of the port-Hamiltonian systems 
simulation technique is possible with minor efforts. More precisely, two different strategies can be adopted:

1) Consider the port-Hamiltonian system as a whole, i.e. all the state variables are included and the different sub-systems are interconnected by means of the skew-symmetric matrix $J$. In this case, the system is characterized by a single energy function and one book-keeping variable;

2) Consider the complete system as the (powerconserving) interconnection of simpler onedimensional sub-systems. In this way, energy functions are separated as well as the bookkeeping variables.

Even if stability is preserved in both cases, the first approach results in "smoother" results, in particular when faulty conditions occur. It is important to note that the proposed approach deeply differs from the socalled Passivity Observer/Passivity Controller (PO/PC) [22], even if both are based on energy considerations and aim at maintaining the passivity of the system. The main difference is that, in our case, the state of the system is properly changed by taking into account the injected power and the system dynamics, and not by considering only a dissipative effect at the input and/or output port.

\section{Passive Interconnection of Systems}

Two novel techniques are now presented for the powerconserving interconnection of digital systems running at different frequencies (see Sect. 4.1), and of continuoustime system (e.g. a haptic interface) with a discrete-time one (e.g. the virtual environment). The starting point is the Passivity Observer / Passivity Controller paradigm, employed both in haptics and in telemanipulation, [9], [10], [16], [23], [24], [25]. The basic idea is to determine if energy is generated at the interconnection and, in such case, to dissipate it in the next iterations of the algorithm. In order to render the simulated system as close as possible to the real one, the energy is not dissipated in one iteration only, but rather the correction is made in more sampling periods, avoiding in this manner the "spikes" generated by traditional PO/PC-based schemes. In any case, the correction is possible by properly changing the force or velocity signals at the interconnection ports.

It is important to note that all the proposed schemes are not able to preserve passivity a priori, but only to make the energy error bounded. In fact, since the actual energy is not precisely known due to the delay caused by the up-down sampling (when two systems simulated at different frequencies are interconnected), or by the time discretization of continuous-time signals (while acquiring the haptic interface joint position), it is not possible to compute the exact energy balance at the end of each sample period, and then a relatively small energy error must be tolerated.

\subsection{Multi-rate port-Hamiltonian systems}

In order to maintain passivity, a novel component that acts as an interface between the fast system, with sample

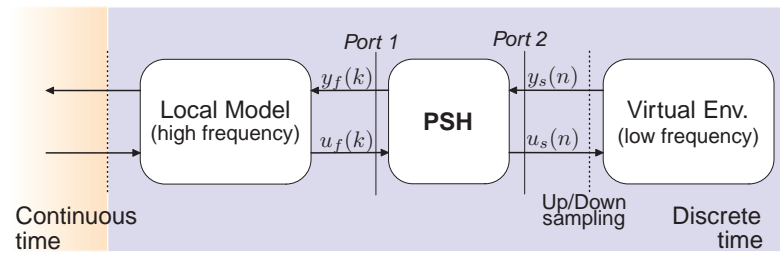

Fig. 7. Passive Sample and Hold (PSH) element, connecting high and low frequencies systems.

period $T_{f}$, and the slow system, with sample period $T_{s}$, is introduced. For simplicity, it is assumed that $T_{s} / T_{f}=N$, with $N \in \mathbb{N}$, and that the two systems are synchronized.

This element, called here Passive Sample and Hold (PSH), is a digital dynamical system running at $T_{f}$. As shown in Fig. 7, it has two inputs, $u_{f}(k)$ and $y_{s}(n)$, and two outputs, $u_{s}(n)$ and $y_{f}(k)$. The pairs $\left(u_{f}, y_{f}\right)$ and $\left(u_{s}, y_{s}\right)$ define two power ports in which $\left(u_{s}, y_{s}\right)$ is updated every $N$ steps because it is interconnected to the slow system. Without loss of generality, scalar power variables are assumed. In case of a system with $n$ inputs and outputs, each pair can be treated separately. The discrete time steps are indicated with $k$ (fast) and $n$ (slow), where $n=\lfloor k / N\rfloor$, and \lfloor\rfloor is the integer part operator. The variation of the energy error $\Delta H_{\mathrm{E}}(n)$ in $\left[n T_{s},(n+1) T_{s}\right)$ is then given by:

$$
\begin{aligned}
& \Delta H_{\mathrm{E}}(n)= \\
& =T_{s} \cdot\left(\frac{1}{N} \sum_{k=n N}^{(n+1) N-1}\left[y_{f}(k) u_{f}(k)\right]-u_{s}(n) y_{s}(n)\right)
\end{aligned}
$$

and, from (13), the energy error $H_{\mathrm{E}}(n)$ at time $t=n T$ is given by

$$
H_{\mathrm{E}}(n)=H_{\mathrm{E}}(n-1)+\Delta H_{\mathrm{E}}(n-1), \quad H_{\mathrm{E}}(0)=0
$$

The outputs of the PSH are then defined as follows:

$$
\begin{aligned}
& y_{f}(k)=y_{s}(n) \quad \forall k \in[n N,(n+1) N-1] \\
& u_{s}(n)=u_{f}(n N)+\frac{\Delta P(n)}{y_{s}(n)}
\end{aligned}
$$

where $\Delta P(n)$ is chosen to bring $H_{\mathrm{E}}(n)$ to zero. This is the key point. Differently from a Passivity Controller, $\Delta P(n)$ is selected in such a way that the difference between $u_{s}(n)$ and $u_{f}(k N)$ is bounded, which means that:

$$
\left|u_{s}(n)-u_{s}(n-1)\right| \leq\left|u_{f}(n N)-u_{s}(N(n-1))\right|
$$

Roughly speaking, the variation of $u_{s}(n)$ with respect to the previous output value $u_{s}(n-1)$ must be less than the difference between $u_{f}(n N)$, which would be the previous value of $u_{s}(n-1)$ in case the PSH was not present, and $u_{s}(n-1)$. By replacing (16) in (17), one obtains

$$
\begin{aligned}
\left|\frac{\Delta P(n)}{y_{s}(n)}+u_{f}(n N)-u_{s}(n-1)\right| & \\
\leq & \left|u_{f}(n N)-u_{s}(n-1)\right|
\end{aligned}
$$




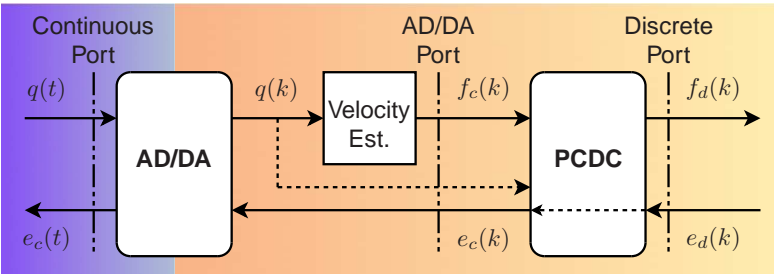

Fig. 8. Interconnection of continuous and discrete time systems by means of AD/DA converter and the Passive Continuous Discrete time Connector (PCDC).

Thanks to the inverse triangle inequality ||$a|-| b|| \leq \mid a+$ $b \mid$, from (18) the following condition can be obtained:

$$
|\Delta P(n)| \leq 2\left|u_{f}(n N)-u_{s}(n-1)\right|\left|y_{s}(n)\right|
$$

This relation is instrumental for overcoming one of the most relevant issues in $P O / P C$-based control schemes. In this case, if $y_{s}(n)$ goes to zero, the corrective term $\Delta P(n) / y_{s}(n)$ of (16) does not diverge since it is bounded by (19). On the other hand, $\Delta P(n)$ has to be chosen in such a way that the energy error $H_{\mathrm{E}}(n)$ goes to zero. For this reason, the PSH corrects (at most) all the energy error accumulated in a step of length $T_{s}$. In particular, $\Delta P(n)$ is chosen so that

$$
|\Delta P(n)| T_{s} \leq\left|H_{\mathrm{E}}(n)\right| \quad \operatorname{sign} \Delta P(n)=\operatorname{sign} H_{\mathrm{E}}(n)
$$

A fast convergence of $H_{\mathrm{E}}(n)$ to zero can be achieved by choosing $|\Delta P(n)|$ equal the maximum value allowed by the bounds (19) and (20). The proposed procedure is then summarized in the following algorithm, where the quantity $H_{\mathrm{E}}(n) / T_{s}$ is used instead of $H_{\mathrm{E}}(n)$ for the sake of convenience:

Algorithm 3 (Passive sample and Hold):

1) Impose $y_{f}(k)=y_{s}(n)$;

2) Compute $\Delta P_{0}=2\left|u_{f}(n N)-u_{s}(n-1)\right|\left|y_{s}(n)\right|$;

3) Compute $\Delta P(n)=\operatorname{sign} H_{\mathrm{E}}(k) \min \left(\Delta P_{0}, H_{\mathrm{E}}(n) / T_{s}\right)$;

4) Compute $u_{s}(n)=u_{f}(n N)+\Delta P(n) / y_{s}(n)$;

5) Compute $H_{\mathrm{E}}(n+1) / T_{s}$, via relation (14).

In step 4), when $y_{s}(n) \simeq 0$, we define $u_{s}(n)=u_{f}(n N)$ to avoid computational problems, even if $\Delta P(n) / y_{s}(n)$ is bounded when $y_{s}(n) \rightarrow 0$ because of (19). Moreover, if the $n$-th iteration of the algorithm is considered, steps 1) to 4) are executed in $t=n T_{s}=k T_{f}$, while step 5) cannot be executed before $t=(n+1) T_{s}-T_{f}=[(n+1) N-$ $1] T_{f}$, since all the values $y_{f}(k), k \in[n N,(n+1) N-1]$ are required to compute $\Delta H_{\mathrm{E}}(n)$. Due to the fact that the PSH requires $N$ inputs from the "fast" system to compute its output, it must be executed at the fastest sampling frequency. Clearly, the output of the PSH can be updated at slow frequency since it is used by the "slow" system, as shown in Fig. 7.

\subsection{Continuous-discrete time connection}

The same philosophy behind the PSH illustrated in the previous section is the starting point for the energy consistent interconnection of haptic interfaces (with position sensors) and passive virtual environment, that are supposed to be described in port-Hamiltonian form. For the sake of completeness, this last assumption is not strictly necessary, since only the energy function associated with the virtual environment is required for the procedure to work. It is assumed that the virtual environment is characterized by an impedance causality, i.e. the position (or velocity) of the haptic interface is the input of the control system, while the interaction force is its output. Due to the fact that usually only position measurements $q(k)$ are available, the (input) velocity must be deduced from position information. With reference to Fig. 8, the two-port system between the "Continuous Port" and the "AD/DA Port" is now taken into account. The interconnection is power conserving if the energy $\Delta H_{\mathrm{ADDA}}(k)$, generated or dissipated in a sample period, is equal to zero. This means that

$$
\begin{aligned}
& \Delta H_{\mathrm{ADDA}}(k)= \\
& \quad=\int_{k T}^{(k+1) T} e_{c}(\tau) f_{\text {cont }}(\tau) \mathrm{d} \tau-T e_{c}(k) f_{c}(k)=0
\end{aligned}
$$

where $f_{\text {cont }}(t)$ is the velocity of the continuous-time system (i.e. on the haptic interface) and $e_{c}(t)$ is the force imposed to the continuous-time system. This force is equal to $e_{c}(k)$ and is generated by the digital controller. If $f_{a}(k)$ denotes the average velocity of the haptic interface in $T$, we have that

$$
\begin{aligned}
& f_{a}(k)=\int_{k T}^{(k+1) T} f_{\text {cont }}(\tau) \mathrm{d} \tau=\frac{q(k+1)-q(k)}{T} \\
& e_{c}(t)=e_{c}(k) \quad \forall t \in[k T,(k+1) T)
\end{aligned}
$$

which implies that

$$
\Delta H_{\mathrm{ADDA}}(k)=T e_{c}(k)\left(\frac{q(k+1)-q(k)}{T}-f_{c}(k)\right)
$$

From (24), it is clear that (21) holds if the estimated velocity $f_{c}(k)$ is chosen as

$$
f_{c}(k)=\frac{q(k+1)-q(k)}{T}
$$

As pointed out in [16], it is not possible to compute (25) in real-time, since the position $q(k+1)$ is not available at time $t=k T$. On the other hand, if the discrete derivative is used to estimate the velocity, i.e.:

$$
f_{c}(k)=\frac{q(k)-q(k-1)}{T}
$$

it is clear from (24) that (21) does not hold anymore.

This problem can be solved by following the same rationale behind the technique proposed in Sect. 4.1 for multi-rate systems. A novel component, called Passive Continuous Discrete time Connector (PCDC), is introduced between the controller and the digital converter. As reported in Fig. 8, the PCDC has two inputs, the (generalized) force coming from the discrete time controller $e_{d}(k)$ and the sampled positions $q(k)$ of the haptic interface, 
and two outputs, the (generalized) velocity $f_{d}(k)$ and the force imposed to the haptic interface $e_{c}(k)$. Similarly to the PSH, the PCDC keeps track of the energy generated or dissipated between the "continuous port" and the "discrete port" at time $t=k T$

$$
H_{\mathrm{E}}(k)=H_{\mathrm{E}}(k-1)+\Delta H_{\mathrm{E}}(k-1), \quad H_{\mathrm{E}}(0)=0
$$

In (27), $\Delta H_{\mathrm{E}}(k)$ is the variation of energy error in the time interval $[k T,(k+1) T)$, given by:

$$
\Delta H_{\mathrm{E}}(k)=\int_{n T}^{(n+1) T} e_{c}(\tau) f_{c}(\tau) d \tau-T e_{d}(k) f_{d}(k)
$$

The PCDC outputs are then chosen as follows (see the similarities with (15) and (16) for the PSH):

$$
e_{c}(k)=e_{d}(k) \quad f_{d}(k)=f_{c}(k)+\Delta P(k) / e_{d}(k)
$$

From (22), (23), and (28) we have that:

$$
\Delta H_{\mathrm{E}}(k)=T e_{d}(k)\left(\frac{q(k+1)-q(k)}{T}-f_{d}(k)\right)
$$

in which $\Delta P(k) / e_{d}(k)$ is an additional term to be applied to the "reference" velocity $f_{c}(k)$. As in the case of the PSH, $\Delta P(k)$ is bounded by the maximum allowed correction on $f_{d}(k)$ :

$$
\left|f_{d}(k)-f_{d}(k-1)\right| \leq\left|f_{c}(k)-f_{c}(k-1)\right|
$$

Note 3: Thanks to (31), the amplitude of the maximum output variation $\left|f_{d}(k)-f_{d}(k-1)\right|$ is bounded by the input variation. This choice is different from (17) in case of PSH because experimental tests proved that the system has unsatisfactory behavior in the latter case. In fact, (31) is a stricter bound than (17).

From (29) and (31), we have that

$$
\left|f_{c}(k)+\frac{\Delta P(k)}{e_{d}(k)}-f_{d}(k-1)\right| \leq\left|f_{c}(k)-f_{c}(k-1)\right|
$$

and then

$$
\begin{aligned}
&\left|\frac{\Delta P(k)}{e_{d}(k)}\right| \leq\left|f_{c}(k)-f_{c}(k-1)\right| \\
&+ \\
&+\left|f_{c}(k)-f_{d}(k-1)\right|
\end{aligned}
$$

from the inverse triangular inequality. This condition provides an upper bound on $|\Delta P(k)|$. Finally, in a sample period, the quantity $T \Delta P(k)$ must not exceed the energy error observed by the PCDC, and the sign of $\Delta P(k)$ must be chosen in order to minimize the energy error $H_{\mathrm{E}}(k)$, which leads to the following conditions:

$$
T \Delta P(k) \leq\left|H_{\mathrm{E}}(k)\right| \quad \operatorname{sign} \Delta P(k)=\operatorname{sign} H_{\mathrm{E}}(k)
$$

The procedure can be easily summarized and implemented by the following algorithm:

Algorithm 4 (PCDC):

1) Compute $H_{\mathrm{E}}(k)$, via relations (27) and (30);

2) Impose $e_{c}(k)=e_{d}(k)$;

3) Compute $f_{c}(k)=(q(k)-q(k-1)) / T$;
4) Compute $P_{0}=e_{d}(k)\left(\left|f_{c}(k)-f_{c}(k-1)\right|+\left|f_{c}(k)-f_{d}(k-1)\right|\right) ;$

5) Compute $\Delta P(k)=\operatorname{sign} H_{\mathrm{E}}(k) \min \left(P_{0}, H_{\mathrm{E}}(k) / T\right)$;

6) Compute $f_{d}(k)=f_{c}(k)+\Delta P(k) / e_{d}(k)$.

A drawback of the approach is the position drift due to the correction operated on the velocity. In any case, since the average velocity can only be estimated, a position drift afflicts the system independently from the PCDC. The choice of modifying the velocity information towards the virtual environment in (29) is a consequence of the fact that it is not possible to modify the force feedback for the haptic interface. This is because the average velocity of the haptic device is not available and can be estimated only with a delay of a sample period. On the other hand, the force signal is perfectly known.

Before concluding, it is important to point out that:

- In case of multi-port systems, the PSH and the PCDC elements must be replicated for each pair of input-output signal since each power port is treated separately one from the others. This means that a PCDC is needed for each actuated degree of freedom of the haptic interface and a PSH for each power port subjected to up-down sapling.

- The estimated velocity $f_{c}(k)$, employed in (29), can be computed by means of more precise methods (e.g. state variable filters), instead of relying on the discrete derivative (26).

\section{VALIDATION AND SIMULATION}

Simulation results showing the behavior of the previously introduced algorithms are now presented. In particular, in Sect. 5.1, two different implementations of the same system are presented to illustrate the methodology of Sect. 3. The mass spring-system is treated as a benchmark. Since the most critical situation in terms of (numerical) stability is the simulation of marginally passive systems, an undamped system is considered, along with its damped counterpart. These two systems are implemented in two different manners each:

- As a single port-Hamiltonian system with two state variables;

- As the interconnection of two port-Hamiltonian systems modeling the mass and the spring separately.

In Sect. 5.2, the result of different simulations of a multirate system are compared. To properly evaluate the achieved performances, an index based on the energy error of the PSH is used as benchmark for a system composed by a spring (fast system), a mass (slow system), and the PSH that connects each other. Finally, in Sect. 5.3, the use of an unilateral spring and of the PCDC element is reported.

\subsection{Simulation of single-rate systems}

This section reports the simulation results of a single rate mass-spring system. The spring stiffness is $k=5000 \mathrm{~N} / \mathrm{m}$, 


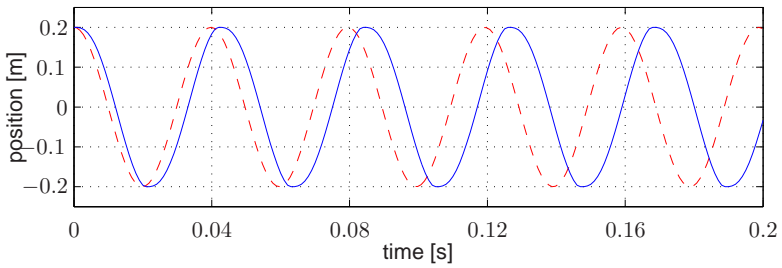

(a) 2-state variables system

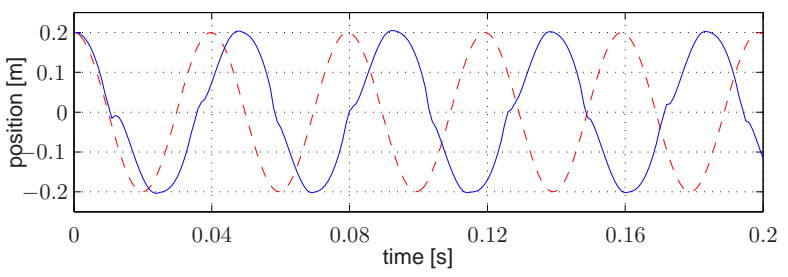

(b) Two 1-state variable systems

Fig. 9. Position evolution of a single-rate, undamped mass-spring system simulated as a single system (a), or two interconnected systems (b). The solid line is the mass position obtained from Algorithm 1, while the dashed line is the reference position.

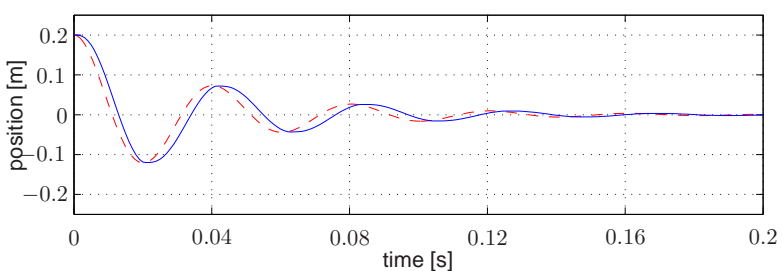

(a) 2-state variables system

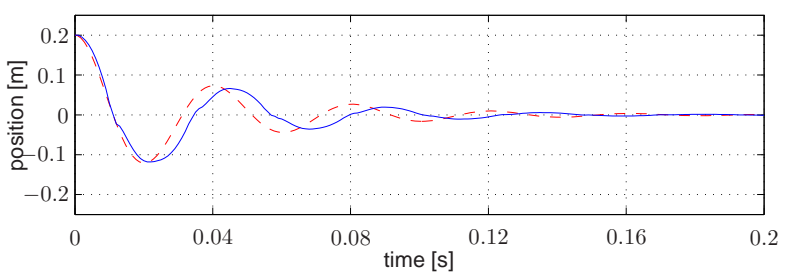

(b) Two 1-state variable systems

Fig. 10. Position evolution of a single-rate, damper-massspring system simulated as a single system (a), or two interconnected systems (b). The solid line is the mass position obtained from Algorithm 1, while the dashed line is the reference position.

the mass is $m=0.2 \mathrm{~kg}$ and, with reference to the system of Fig. 10, a damping coefficient $b=10 \mathrm{~N} \cdot \mathrm{s} / \mathrm{m}$ has been adopted. Moreover, the systems are autonomous, i.e. no external forces are acting on the masses, and are characterized by an initial position $q_{0}=0.2 \mathrm{~m}$, with zero velocity (i.e. null initial momentum). Finally, the integration time step is $T=1 \mathrm{~ms}$. These are the same conditions of the simulations of Example 2 and reported in Fig. 3. Fig. 9(a) and 10(a) present the time evolution of the position of the systems implemented with two state variables, while Fig. 9(b) and 10(b) refer to the systems resulting from the interconnection of two onedimensional sub-systems, i.e. the spring and the mass are simulated separately, and the output velocity of the mass is the input of the spring, while the force generated by the spring is used as input of the mass.

The solid lines in Fig. 9 represent the time evolution of the mass position when Algorithm 1 is used as integration method, while the dashed lines indicate the ideal simulation results, obtained with a variable step integration method, i.e. ODE45. In Fig. 9(b) and 10(b), the effects of energy leaps when the position 'crosses' the zero value can be noticed since this fact is responsible for a "discontinuity". This effect is not present in the two-state system without damping, see Fig. 9(a), because fault conditions do not occur. This is due to the fact that the total energy, which is the sum of the kinetic energy of the mass and of the potential energy of the spring, is constant and always different from zero. Even in case of damping, energy leaps (rarely) appear only when the system oscillates around zero. In this case, however, they have no relevant effects on the system dynamics, as can be seen in Fig. 10(a). From these examples it appears that, as a design guideline, it is better to implement a virtual environment by means of a dynamical system with "more state variables", so that fault conditions could take place only when at least a significant portion of the environment is "at rest" (i.e. the total energy is close to its minimum). With this choice, the user does not feel these occasional "bumps" in the simulated environment and experiences a more realistic haptic rendering. Moreover, both systems present a significant change in their natural oscillation frequency due to the choice of the update strategy, but this frequency variation is scarcely perceived by the user and it cannot be considered as a significant drawback.

\subsection{Simulation of multi-rate system}

In order to qualitatively understand under which conditions the behavior of the Passive Sample and Hold is "acceptable", several simulations of a multi-rate system have been performed with different choices in the parameters. The benchmark is the simple mass-spring system in which the mass is simulated as a slow process (integration time step $T_{s}$ ), while the spring is simulated as a fast one (integration time step $T_{f}$ ). Performances have been compared by introducing the numerical index $H_{\text {norm }}=H_{\text {mean }} / H_{\text {sys }}$. Here, $H_{\text {mean }}$ is the average value of the energy error $H_{\mathrm{E}}(n)$ (see (14)) taken over a sufficiently long time interval so that a steady-state value has been reached, while $H_{\text {sys }}$ is the initial energy of the system. As far as the example under study is concerned, since the system is not dissipative, it is clear that $H_{\text {sys }}$ is constant and then $H_{\text {norm }}$ indicates how much energy is inserted into the system. It's worth noticing that, even if ideally the index should assume a value close to zero, the system remains stable if $H_{\text {norm }}$ has a finite value because the PSH always injects a finite quantity of energy. It is the authors' opinion that the parameters should be chosen in such a way that $H_{\text {norm }}$ is less than 0.2 for the simulated system to perform similarly to the real one. 


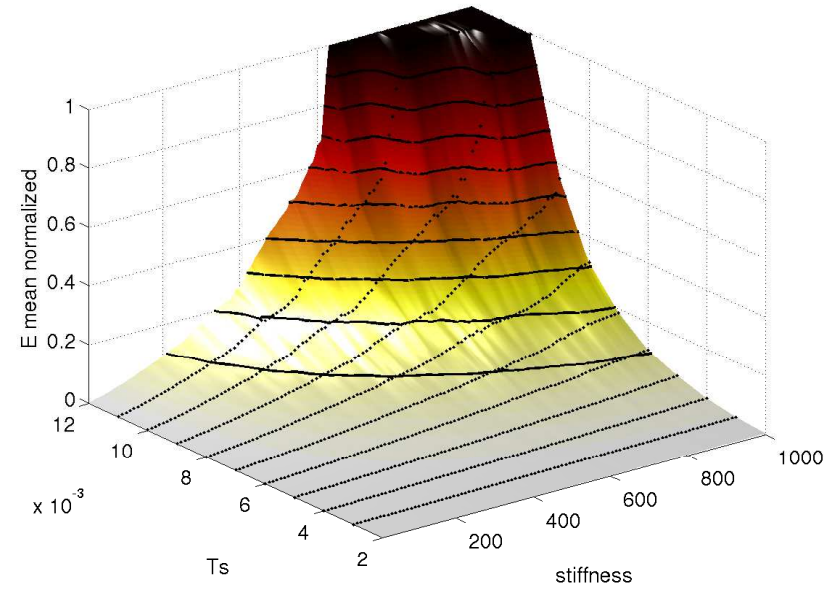

(a) Variable stiffness ( $k$ expressed in $\mathrm{N} / \mathrm{m}$ ) and $m=0.2 \mathrm{~kg}$

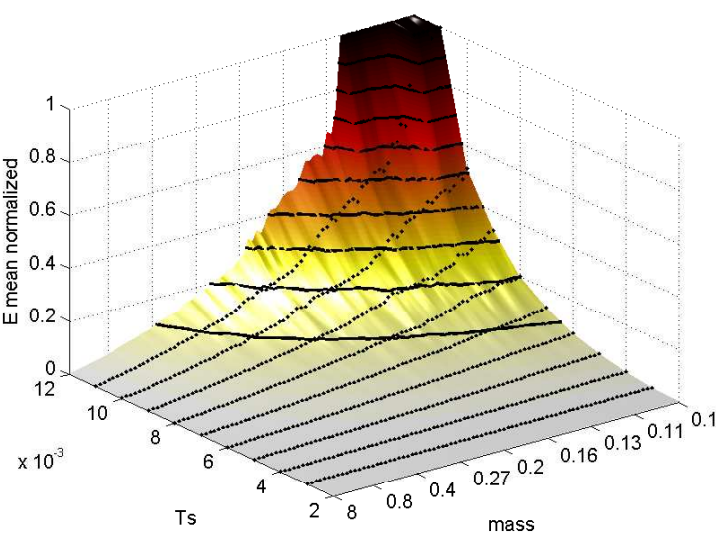

(b) Variable mass ( $m$ expressed in $\mathrm{kg}$ ) and $k=400 \mathrm{~N} / \mathrm{m}$

Fig. 11. $H_{\text {norm }}$ for different choices of $k, m$ and $T_{s}$ in a multi-rate mass-spring system.

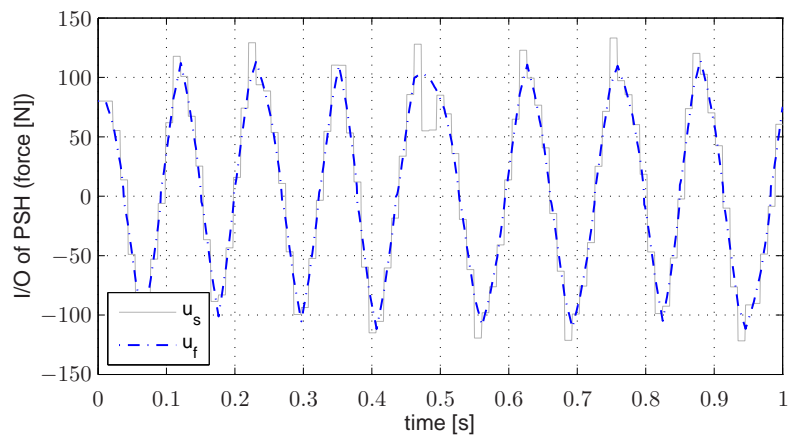

(a) $T_{s}=11 \mathrm{~ms}, k=400 \mathrm{~N} / \mathrm{m}$

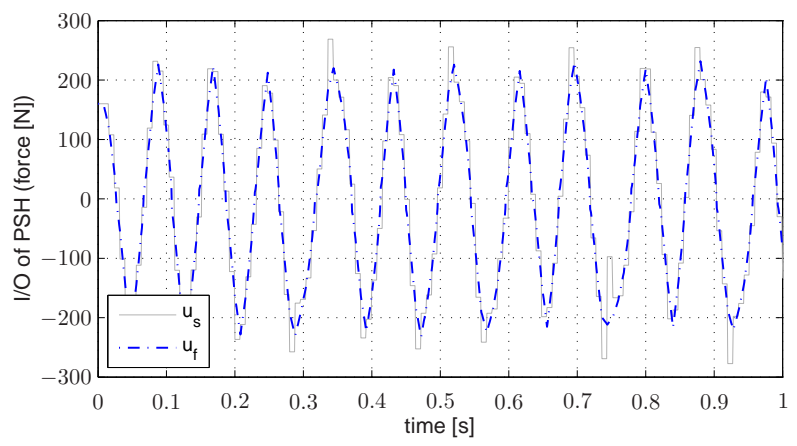

(b) $T_{s}=8 \mathrm{~ms}, k=800 \mathrm{~N} / \mathrm{m}$

Fig. 12. Evolution of $u_{f}$ and $u_{s}$ for a PSH inserted in a multi-rate mass-spring system, with different choices of $T_{s}$ and $k$, and with $T_{f}=1 \mathrm{~ms}, m=0.1 \mathrm{~kg}$.

Two different sets of simulations have been performed. In the first one, the mass has been set equal to $m=0.2 \mathrm{~kg}$ while the spring stiffness ranges from $k=10 \mathrm{~N} / \mathrm{m}$ to $k=1000 \mathrm{~N} / \mathrm{m}$. In the second set of simulations, the spring stiffness is set to $k=400 \mathrm{~N} / \mathrm{m}$ and the value of the mass ranges from $m=0.1 \mathrm{~kg}$ to $m=8 \mathrm{~kg}$. In both cases, the initial velocity is zero, the initial position is $q_{0}=0.2 \mathrm{~m}, T_{f}=1 \mathrm{~ms}$, and $T_{s} \in\{2,3, \cdots, 12\} \mathrm{ms}$. The values assumed by $H_{\text {norm }}$ in these different conditions are reported in Fig. 11(a) (constant mass, variable stiffness) and Fig. 11(b) (constant stiffness, variable mass). For a better comparison of the results, the mass axis in Fig. 11(b) is shown for decreasing values since similar changes in $k$ or $m$ generate opposite effects to the energy function (4) of the mass/spring system.

In order to show the behavior of the PSH, the results of two different experiments for which $H_{\text {norm }}=0.2$ are also illustrated. In Fig. 12, the time evolution of $u_{f}$ (dashed lines) and $u_{s}$ (solid lines), see (16), for different choices of system parameters are reported. In particular, Fig. 12(a) shows the system behavior when $m=0.2 \mathrm{~kg}$, $k=400 \mathrm{~N} / \mathrm{m}$ and $T_{s}=11 \mathrm{~ms}$, while Fig. 12(b) when $m=0.2 \mathrm{~kg}, k=800 \mathrm{~N} / \mathrm{m}, T_{s}=T_{s}=8 \mathrm{~ms}$. Note that in both cases the controller output roughly follows the input and no position drift occurs since the $u_{s}$ and $u_{f}$ are forces. Moreover, the system is marginally stable, with oscillations greater than the continuous time counterpart because of the energy error.

\subsection{Simulation of the bouncing ball}

In order to show the features of the PCDC presented in Sect. 4.2 and of Algorithm 2, which models an unilateral spring, the simulation of a bouncing ball is now reported and discussed. The ball has the mass $m=0.2 \mathrm{~kg}$ and, under the effect of the gravity field, falls on a virtual surface (wall) with stiffness $k=5000 \mathrm{~N} / \mathrm{m}$ from a height of $0.2 \mathrm{~m}$. The system is not dissipative. The mass is treated as a continuous time system, interconnected by means of the PCDC element to the discrete time model, executed at $1 \mathrm{kHz}$. The position of the mass is directly connected to the spring and acts as reset condition.

Fig. 13(a) and 13(b) show that the system is stable. More in detail, Fig. 13(b) reveals that a small amount of energy is generated during the short impact period in which the system is not able to dissipate the power error. The total energy is calculated as the kinetic plus potential energy of the continuous time mass, summed to the 


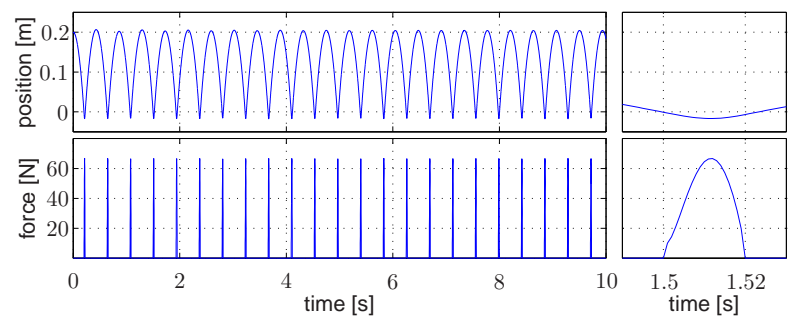

(a) Mass position and force exerted by the unilateral spring.

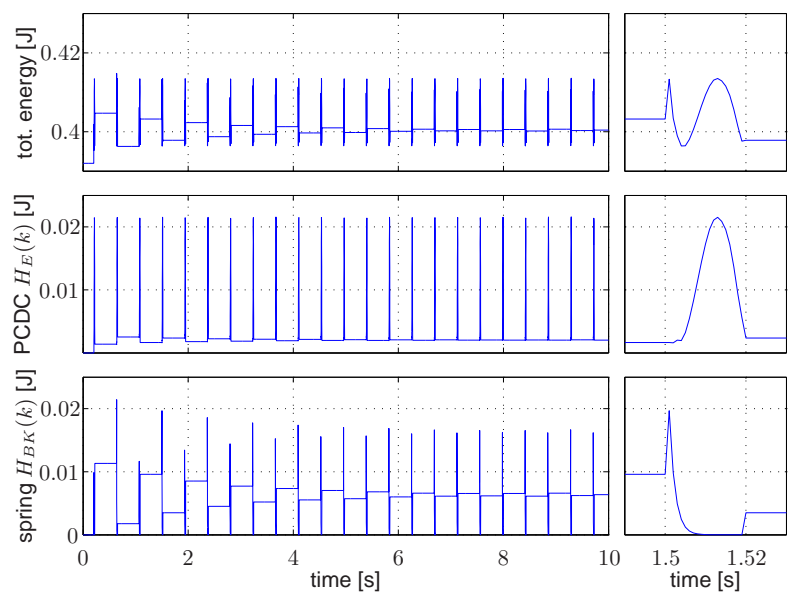

(b) Total system energy (top), PCDC energy (middle), and spring generated energy (bottom).

Fig. 13. Simulation of a bouncing ball. A mass (continuous time system) under the effect of the gravity field is interconnected to an unilateral spring (discrete time system) by means of the PCDC.

potential energy of the spring. The energy generated is the sum of the energy in the interconnection element and the bookkeeping variable of the spring. The difference between the total energy and the generated energy is constant. It is possible to verify that this system can become unstable for high values of $k$, when PCDC and passive simulation of the spring are not able to bring to zero (or at least bound) the generated energy.

\section{EXPERIMENTS}

The techniques described in the previous sections have been tested also by means of an extensive experimental activity. The haptic interface in this case consists of a linear motor LinMot P01-23Sx80 actuated by the servo controller LinMot E210-VF. The motor is equipped with an encoder characterized by a resolution of $1 \mu \mathrm{m}$. Moreover, a high sensitivity load cell is placed between the motor slider and the user handle. The control is implemented on a Pentium IV PC equipped with a Sensoray 626 data acquisition board. The OS is RTAILinux based on a Debian distribution. The real time I/O support for the acquisition board is provided by Comedi drivers. The sample period is $T=1 \mathrm{~ms}$. To compensate the friction of the slider, a control scheme based on a momentum observer has been employed, [26], [27]. The sub-system composed by the controller and the linear

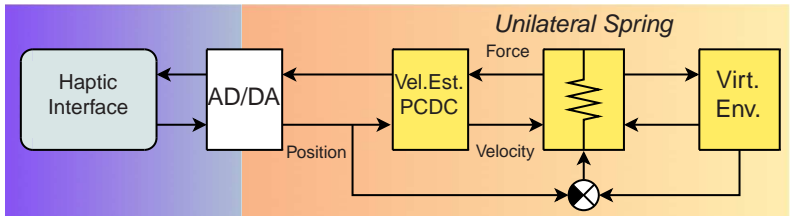

(a) Virtual wall experiment.

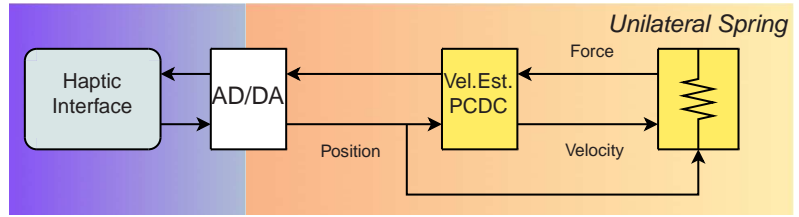

(b) Virtual environment experiment.

Fig. 14. Schematic diagrams of the experiments.

motor can be regarded as a mass-damper system with mass $m=0.150 \mathrm{~kg}$ and a damping coefficient $b=2 \mathrm{~N} \cdot \mathrm{s} / \mathrm{m}$.

This setup has been employed to carry out two sets of experiments. In the first one, the simulation of a virtual wall characterized by a stiffness of $20 \mathrm{kN} / \mathrm{m}$ has been implemented. The functional diagram of the experiment is reported in Fig. 14(a). Note the position signals entering the unilateral spring in order to trigger the reset. The data collected during various interactions with the virtual wall have been reported in Fig. 15.

In the first upper plot of Fig. 15(a), the haptic interface position and spring elongation are shown, while in the lower one the forces generated by the spring and measured by the load cell are reported. These forces are slightly different due to the dynamical effects of the motor slider. In Fig. 15(b), the energy stored in the spring and the total energy error (the sum of the bookkeeping variable and of the PCDC energy) are compared. Since the unilateral spring is the only energy storing element, energy is different from zero only during contact phases (positive HI positions). The second experiment concerns the interaction with an object modeled as a mass-springdamper system $(m=10 \mathrm{~kg}, k=100 \mathrm{~N} / \mathrm{m}, b=1 \mathrm{~N} \cdot \mathrm{s} / \mathrm{m})$. In this case, the haptic interface interacts with the virtual environment through a virtual coupling characterized by a stiffness of $10 \mathrm{kN} / \mathrm{m}$, as shown in Fig. 14(b). In Fig. 16(a), the contact force spikes that appear when the haptic interface collides with the environment can be noted, together with the force exerted by the user to compress the virtual object. Finally, in Fig. 16(b), the total energy stored in the virtual coupling and environment, and the total energy error, i.e. the energy error of the PCDC summed to the bookkeeping variables of the virtual coupling and environment systems, are reported.

Before concluding, it is worth mentioning the mechanism behind the flow of energy into the virtual environment. Energy is at first transferred from the human operator towards the virtual environment through the haptic interface (e.g. when he exerts a force on the virtual environment). Then, energy is dissipated by the damping element within the virtual environment, or 


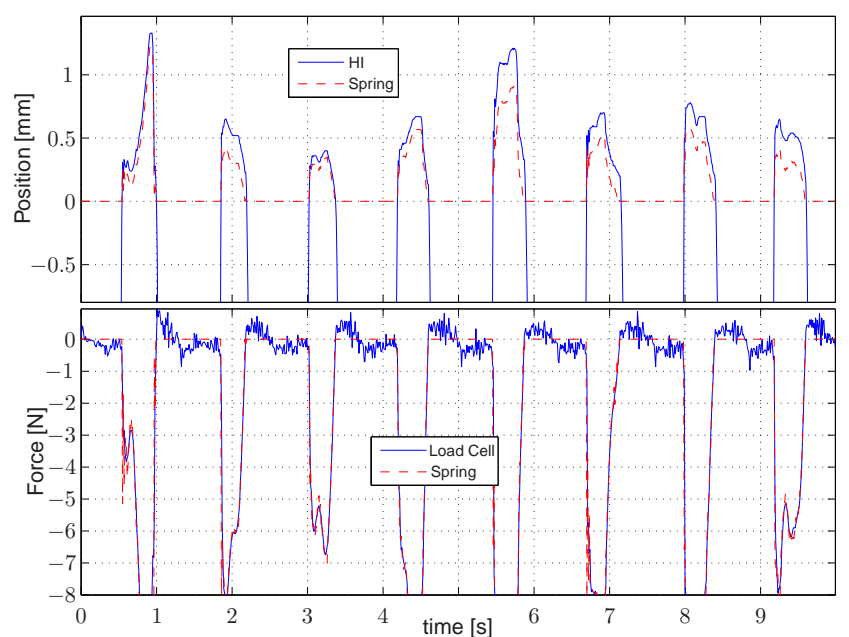

(a) Positions and forces.

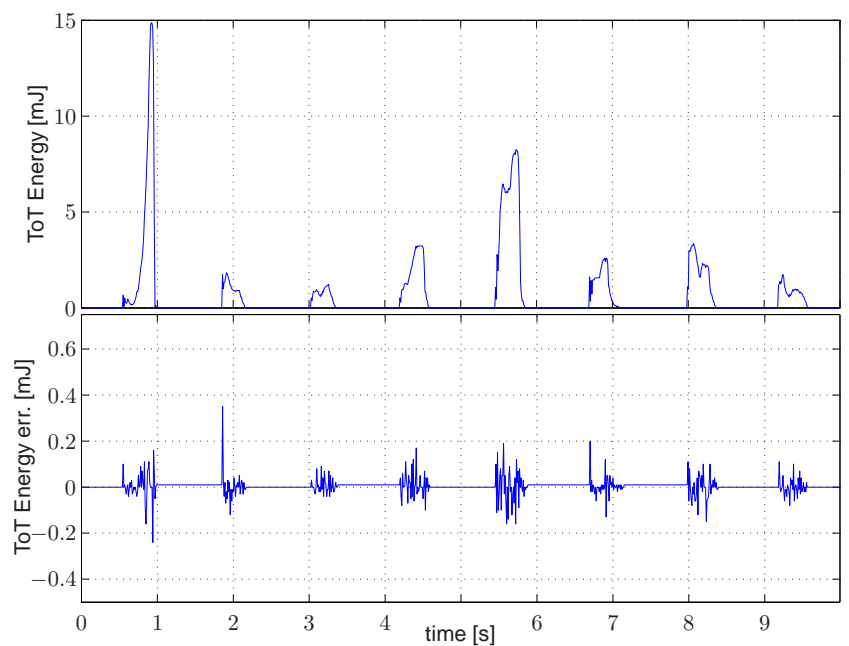

(b) Total energy and total energy error.

Fig. 15. Virtual wall experiment.

transferred back to the human operator. It is clear that the human operator can be treated as a sort of "source of force" applied to the haptic device which can, obviously, absorb or generate energy.

\section{CONCLUSION AND FINAL REMARKS}

This paper deals with different simulation and implementation problems in haptics. At first, the problem of energy consistent simulation of dynamical systems is tackled. A systematic procedure for obtaining a discrete time approximation of a port-Hamiltonian system which satisfies the same energy balance relation of its continuous time counterpart at the end of each sample period is illustrated. The proposed algorithm generalizes previous results since the following features have been added:

- Extension to systems with more state variables;

- Definition of a general method for the energy consistent update of the state;

- Description of a general method for tackling all the faulty conditions and the recovery from energy

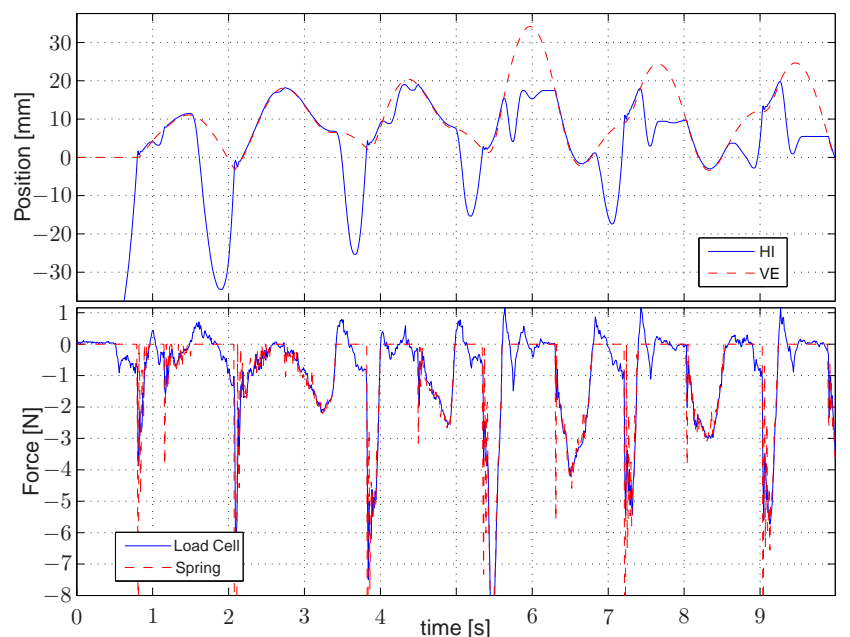

(a) Positions and forces.

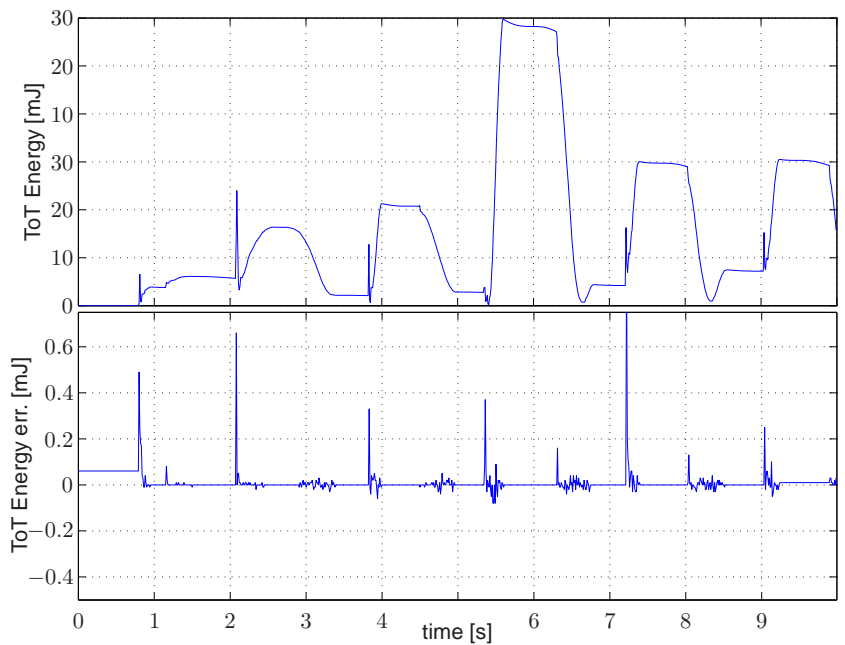

(b) Total energy and total energy error.

Fig. 16. Virtual environment experiment.

errors, in substitution of ad hoc methods to recover from particular fault conditions;

- Further analysis of the management of the faulty condition, aiming at defining new systems, such as the unilateral spring.

Then, in Sect. 4, a novel technique for the power conserving interconnection of digital systems running at different frequencies is illustrated. This methodology is also applied to the case of continuous and discrete time systems interconnection, in order to deal with the power generation effects due to analog/digital conversion. The algorithms have been tested by simulations and experiments with a one dof haptic interface. However, the definition of the maximum energy that the PSH or the PCDC can correct in each sample period is still an open issue. As already pointed out, these upper bounds have been empirically determined, and they can be modified at will. Future results will relate these bounds not only to the input/output signals, but also to the energy error. In this way, these "junction" elements can recover from 
critical situations more easily, thus allowing stronger control actions if the system tends to become unstable.

\section{REFERENCES}

[1] J. E. Colgate and J. M. Brown, "Factors affecting the Z-width of a haptic display," in Proc. of IEEE International Conference on Robotics and Automation, 1994, pp. 3205-3210.

[2] J. J. Abbot and A. M. Okamura, "Effects of position quantization and sampling rate on virtual-wall passivity," IEEE Trans. on Robotics, vol. 21,5, pp. 952 - 964, 2005.

[3] J. G. Park and G. Niemeyer, "Haptic rendering with predictive representation of local geometry," in Proc. of IEEE Haptics Symposium, 2004, pp. 331-338.

[4] J. E. Colgate, P. E. Grafing, M. C. Stanley, and G. Schenkel, "Implementation of stiff virtual walls in force-reflecting interfaces," in Proc. of IEEE Virtual Reality Symposium, 1993, pp. 202-208.

[5] N. Diolaiti, G. Niemeyer, F. Barbagli, and J. K. Salisbury, "A criterion for the passivity of haptic devices," in Proc. of IEEE International Conference on Robotics and Automation, 2005, pp. 2463 2468.

[6] N. Diolaiti, G. Niemeyer, F. Barbagli, J. K. Salisbury, and C. Melchiorri, "The effect of quantization and Coulomb friction on the stability of haptic rendering," in Proc. of IEEE World Haptics Conference, 2005, pp. 273-246.

[7] F. Barbagli, D. Prattichizzo, and J. K. Salisbury, "A multirate approach to haptic interaction with deformable objects single and multipoint contacts," International Journal of Robotics Research, vol. 24, no. 9, pp. 703-715, 2005.

[8] _ "Dynamic local models for stable multi-contact haptic interaction with deformable objects," in Proc. of the 11th Symposium on Haptic Interfaces for Virtual Environment and Teleoperator Systems, 2003, pp. $109-116$.

[9] B. Hannaford and J.-H. Ryu, "Time domain passivity control of haptic interfaces," in Proc. of IEEE International Conference on Robotics and Automation, 2001, pp. 1863-1869.

[10] M. L. McLaughlin, J. P. Hespanha, and G. S. Sukhatme, Touch in Virtual Environments. Pearson Education, 2001

[11] S. Stramigioli, C. Secchi, A. van der Schaft, and C. Fantuzzi, "A novel theory for sample data system passivity," in Proc. of IEEE/RSI International Conference on Intelligent Robots and Systems, 2002.

[12] S. Stramigioli, C. Secchi, and C. Fantuzzi, "Delayed virtual environments: a port-hamiltonian approach," in Proc. of IEEE/RSI International Conference on Intelligent Robots and Systems, 2003.

[13] S. Stramigioli, C. Secchi, A. van der Schaft, and C. Fantuzzi, "Sampled data systems passivity and discrete port-hamiltonian systems," IEEE Trans. on Robotics, vol. 21,4, pp. 574 - 587, 2005.

[14] C. Secchi, S. Stramigioli, and C. Fantuzzi, Control of interactive robotic interfaces: A port-Hamiltonian approach, ser. Springer Tracks in advanced robotics. New York: Springer Verlag, 2007, vol. 29.

[15] G. Borghesan, A. Macchelli, and C. Melchiorri, "Simulation issues in haptics," in Proc. of IEEE International Conference on Robotics and Automation, 10-14 April 2007, pp. 111-116.

[16] B. Hannaford, J.-H. Ryu, and Y. S. Kim, "Sampled- and continuous-time passivity and stability of virtual environments," IEEE Trans. on Robotics, vol. 20,4, pp. 772-776, 2004.

[17] B. M. Maschke and A. J. van der Schaft, "Port controlled Hamiltonian systems: modeling origins and system theoretic properties," in Proc. of the third Conference on nonlinear control systems (NOLCOS), 1992.

[18] — " "Interconnection of systems: the network paradigm," in Proc. 35rd IEEE Conf. on Decision and Control, Dec. 11-13 1996, pp. 207-212.

[19] A. J. van der Schaft, $L_{2}$-Gain and Passivity Techniques in Nonlinear Control, ser. Communication and Control Engineering. Springer Verlag, 2000.

[20] V. Duindam, A. Macchelli, S. Stramigioli, and H. Bruyninckx, Eds., Modeling and Control of Complex Physical Systems. The PortHamiltonian Approach. Springer, 2009.

[21] J. G. de Jalon and E. Bayo, Kinematic and Dynamic Simulation of Multibody Systems: The Real Time Challenge. Secaucus, NJ, USA Springer-Verlag New York, Inc., 1994.

[22] J.-H. Ryu, P. C., B. Hannaford, and G. Hirzinger, "Time domain passivity control with reference energy following," IEEE Trans. on Control Systems Technology, vol. 13, no. 5, pp. 732-742, 2005.
[23] B. Hannaford and J.-H. Ryu, "Time domain passivity control of haptic interfaces," IEEE Trans. on Robotics, vol. 18, pp. 1-10, 2002.

[24] B. Hannaford, J.-H. Ryu, and D.-S. Kwon, "Control of a flexible manipulator with noncollocated feedback: time-domain passivity approach," IEEE Trans. on Robotics, vol. 20, no. 4, pp. 776-780, 2004.

[25] - "Stable teleoperation with time domain passivity control," IEEE Trans. on Robotics, vol. 20, no. 2, pp. 365-373, 2004.

[26] A. De Luca and R. Mattone, "Actuator failuture detection and isolation using generalized momenta," in Proc. of IEEE International Conference on Robotics and Automation, Taipei, Taiwan, 2003, pp. 634-639.

[27] G. Palli and C. Melchiorri, "Non-model based friction and load compensation in linear electric drives," in Proc. 4th Int. Symp. on Motion Control, 2007.

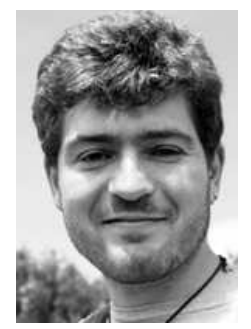

Gianni Borghesan (M'10) received the M.Sc. degree in computer science engineering in July 2004 at the University of Pisa, Pisa, Italy and the Ph.D. degree in June 2008 at the University of Bologna, Bologna, Italy. He as been visiting student at Mechanical Department of the New Jersey Institute of Technology (NJIT), Newark, NJ, USA, and Deutsches Zentrum für Luft- und Raumfahrt (DLR), Munich, Germany, in 2004 and 2007 respectively. He currently holds a PostDoc position at the Department of Electronics, Computer sciences and Systems of the Bologna University. His research activity is centered on haptic interface and telemanipulation control based on passivity criteria, and lately on the modeling and control of tendon actuated robotic hands.

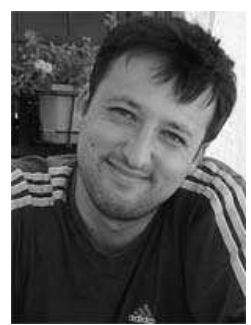

Alessandro Macchelli (M'06) received the M.Sc. degree (cum laude) in computer science engineering in February 2000, and the Ph.D. degree in May 2003, both from the University of Bologna, Bologna, Italy. In 2001, he was appointed Visiting Scholar and, in 2003, got a PostDoc position both at the Department of Applied Mathematics (TW) of the University of Twente, Enschede, The Netherlands. Since 2005 he has been faculty member at the Department of Electronics, Computer Science and Systems of the University of Bologna as Assistant Professor. His research activity is focused on the modelling, simulation and control aspects of finite and infinite dimensional systems within the port-Hamiltonian framework.

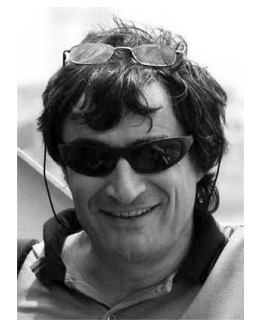

Claudio Melchiorri (M'92 - SM'00) was born on October 23, 1959. He received the Laurea degree in electrical engineering in 1985 and the Ph.D. degree in 1990, both from the University of Bologna, Bologna, Italy. He was appointed Adjunct Associate in Engineering in the Department of Electrical Engineering, University of Florida, Gainesville, in 1988, and Visiting Scientist in the Artificial Intelligence Laboratory, Massachusetts Institute of Technology, Cambridge, for periods in 1990 and 1991. Since 1985, he has been with DEIS, the Department of Electrical Engineering, Computer Science and Systems, University of Bologna, working in the field of robotics and automatic control. He currently holds the position of Full Professor in Robotics at the University of Bologna. His research interests include dexterous robotic manipulation, haptic interfaces, telemanipulation systems, advanced sensors, and nonlinear control. He is the author or coauthor of about 200 scientific papers presented at conferences or published in journals, and of 13 books on digital control and robotics. 